\title{
Social Media Responses to Online Suicide-Related News Articles
}

\author{
Tiana Edwards ${ }^{1}$, Michelle Torok¹, Lauren McGillivray', Trinn Ford², \\ Katherine Mok' ${ }^{1}$ Emily Li ${ }^{1}$, and Mark E. Larsen ${ }^{1}$
}

${ }^{1}$ Black Dog Institute, University of New South Wales, Sydney, Australia

${ }^{2}$ Faculty of Law, University of New South Wales, Sydney, Australia

\begin{abstract}
Background: Responsible media reporting of suicide is important to prevent contagion effects. Online media reporting is increasingly becoming the primary source of news information for many people. Aims: This study aimed to assess compliance with responsible media reporting guidelines, and whether social media responses were associated with compliance. Method: A random sample of Australian digital news articles over a 9-month period were coded for compliance to Mindframe suicide reporting guidelines. Social media responses (number of shares and number of comments) were collected via Facebook. Results: From the sample of 275 articles, articles were compliant with a median of seven of the nine recommendations. Articles compliant with more than seven recommendations were shared more frequently (median: 93 vs. 38 shares, $p=.017$ ) but no difference was observed in the number of comments (median: 0 vs. $0, p=.340$ ). Limitations: Other factors associated with individual events and articles are also likely to contribute to the response on social media. Although no difference in the number of comments was observed, the nature of these comments may differ. Conclusion: Improved understanding of how to maximize dissemination of positive messages may help minimize contagion effects.
\end{abstract}

Keywords: social media, online news, suicide reporting, media guidelines

Media reporting of suicide has the capacity to influence rates of imitation suicide in the weeks following publication of the index event - often referred to as the Werther effect. A systematic review of over 100 studies supported a relationship between media reporting and imitation suicide (Pirkis \& Blood, 2010). The underlying mechanisms for imitation suicide are poorly understood, but social learning theory may help understand it, as most human behavior is learned from others, a suicide is more likely to occur after the individual has learned a method to carry it into effect. For instance, studies have shown a relationship between pro-suicide material available online and increased suicide rates (Colombo, Burnap, Hodorog, \& Scourfield, 2016; Mueller \& Abrutyn, 2015). Likewise, providing information on help-seeking is important to counteract the effect of contagion, known as the Papageno effect (Niederkrotenthaler et al., 2010). The Papageno effect benefits the community via the media's reporting on information about crisis resources, educating the public about mental illness myths and facts, and individuals who have recovered from depression and suicidal ideation (Pirkis \& Blood, 2010; World Health Organization and the International Association for Suicide Prevention, 2017).

Sensationalist news reports contribute toward a biased perception of the influences impacting at-risk individuals (Niederkrotenthaler et al., 2012) and can be manipulated to garner high rates of viewership. The over-reporting of certain populations - such as the elderly, youth, and those with a high social status - misrepresents the actual populations who are most susceptible or at-risk (Gould, Kleinman, Lake, Forman, \& Midle, 2014; Lee, Lee, Hwang, \& Stack, 2014).

The World Health Organization and the International Association for Suicide Prevention (2017) developed evidence-based guidelines for reporting on suicide that many organizations have since used to draft local versions. For example, Mindframe is the Australian guideline for how to report on suicide responsibly (Everymind, 2014). These guidelines recommend emphasizing the importance of telling the whole story with suicide, help-seeking behavior, and positive stories of overcoming suicidal thoughts/ depression. Responsible reporting is assisted by avoiding sensational language, and specific and confronting images that can perpetuate stigma and misconceptions about suicide. Within the Australian context, there are approximately 3,000 deaths by suicide each year with a general upward trend in suicide rate over the past decade (Australian Bureau of Statistics, 2019). Suicide prevention is now a key priority at the federal and state levels (Department of Health, 2017).

Media guidelines should reflect current and emerging best-practice - particularly digital media, which is rapidly becoming the primary source of news consumption, to avoid the potential for harmful contagion. While the use of 
digital news platforms increases, it is unclear how well online media reports conform to Mindframe. Further, given that digital platforms have the potential to reach a greater audience, it is critical that suicide is reported responsibly. Better understanding of social contagion, including how the online community responds to news articles, is important for revising online reporting guidelines and could provide insight into protective strategies, such as online support networks (De Choudhury \& Kiciman, 2017). Accordingly, the objective of this study was to determine the extent to which online news reports by Australian media organizations adhere to Mindframe suicide reporting guidelines, and to assess whether responsible reporting was associated with differences in social media responses.

\section{Method}

This study analyzed a database of suicide-related media articles, collated via Google News searches. Articles over the 9-month period from April to December 2017 were reviewed for eligibility for inclusion in this study. Articles were excluded if they did not discuss a suicide event, an individual's suicide attempt or suicidal ideation, if they described fictional content, or if they were related to terrorism. From the eligible articles, a random 30\% sample from each calendar month were retained and downloaded for analysis.

A coding framework was developed to capture the nine Mindframe principles of responsible media reporting of suicide events. These nine items were operationalized as:

1. Does the story include inappropriate or sensationalist

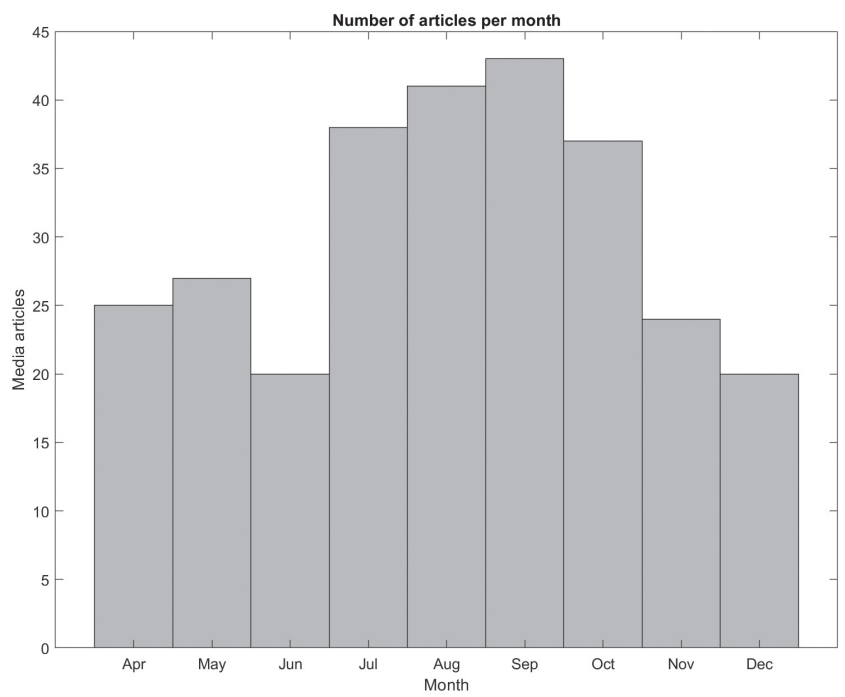

Figure 1. Distribution of coded media articles per month over the study period, 2017 language (e.g., "successful suicide," "commit suicide," "failed suicide," "political suicide")?

2. Is the word suicide used in the headline?

3. Does the story include visual media depicting the suicide scene, memorial, funeral, precise location or method/means?

4. Does the article include a detailed discussion of the method/means used (e.g., steps involved, how means were obtained, exact locations)?

5. Were specific details of a suicide note disclosed?

6. Was it implied that suicide is spontaneous or due to a single event (vs. exploring a range of factors)?

7. Is information about help-seeking or services provided?

8. Were the bereaved interviewed immediately after the death?

9. Are public or online memorials promoted?

Two researchers coded each article, and disagreements were resolved by discussion until consensus was achieved. Each item, except Item 7 on help-seeking information, was then reverse coded $(0=y e s, 1=n o)$, and a total score (0-9) was calculated to represent the overall degree of concordance with responsible reporting guidelines.

Social media responses to the articles on Facebook were examined via the Graph application programming interface (API) to identify the number of times the article had been shared and the total number of comments made in response to these posts. These data were collected in $\mathrm{Au}-$ gust 2018, representing a period of at least 8 months after the initial media report and a time in which further social media activity is unlikely.

Descriptive summary statistics were reported for compliance with each reporting guideline, and the number of compliant recommendations per coded article. Articles with low and high compliance were dichotomized based on the median compliance level, and Wilcoxon rank sum tests were performed to compare the numbers of Facebook shares and Facebook comments for the subgroups. All analyses were conducted using Matlab 8.6. This study was approved by the University of New South Wales Human Research Ethics Committee.

\section{Results}

After screening for eligibility and random sampling, a total of 275 media articles were retained in the analysis set for coding. The distribution of articles over the nine months is shown in Figure 1.

Compliance with each of the nine reporting recommendations was generally good (see Figure 2a). Compliance was lowest with the second guideline (inclusion of the 

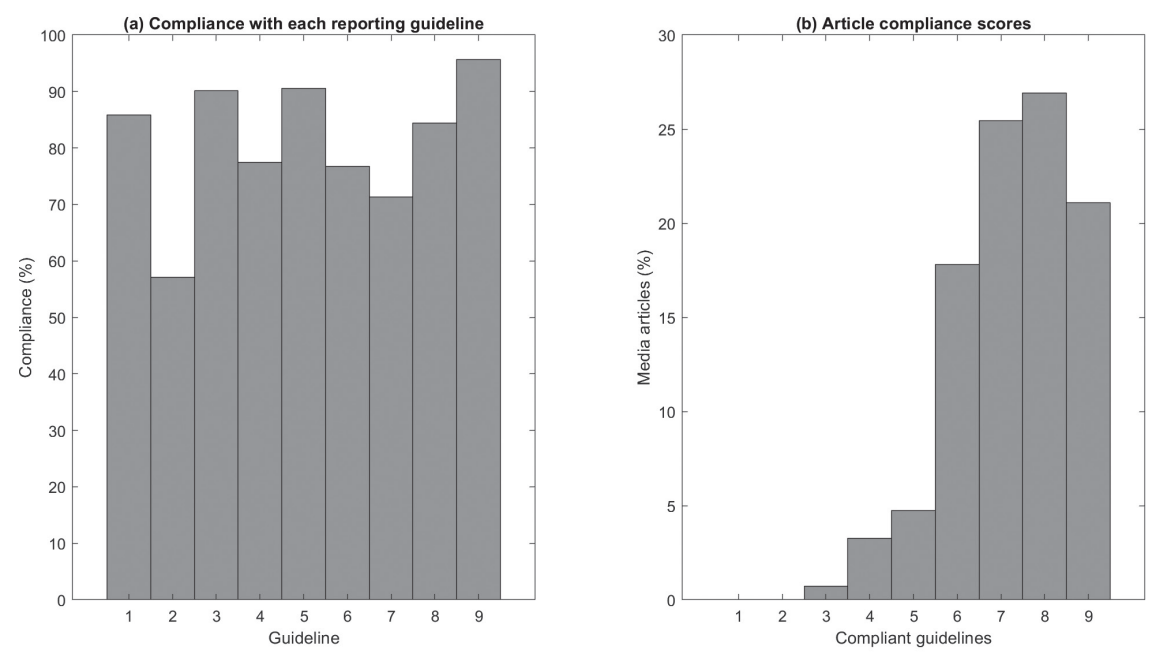

Figure 2. (a) Proportion of coded media articles that were compliant with each of the nine reporting recommendations. (b) Distribution of compliance scores across the coded articles.

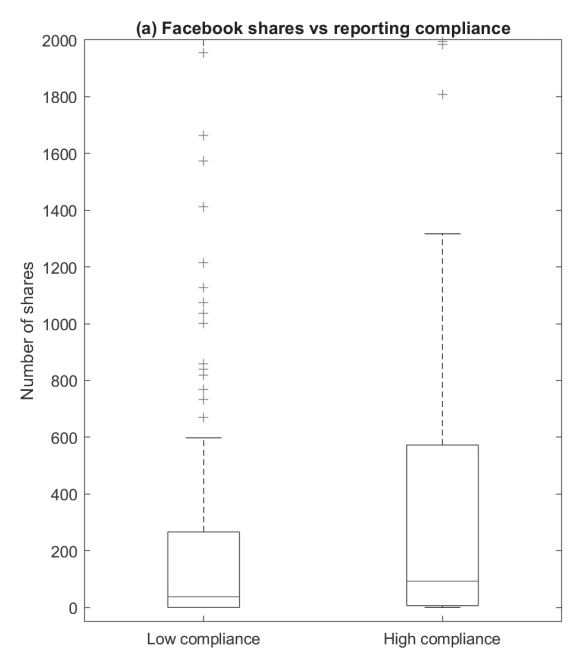

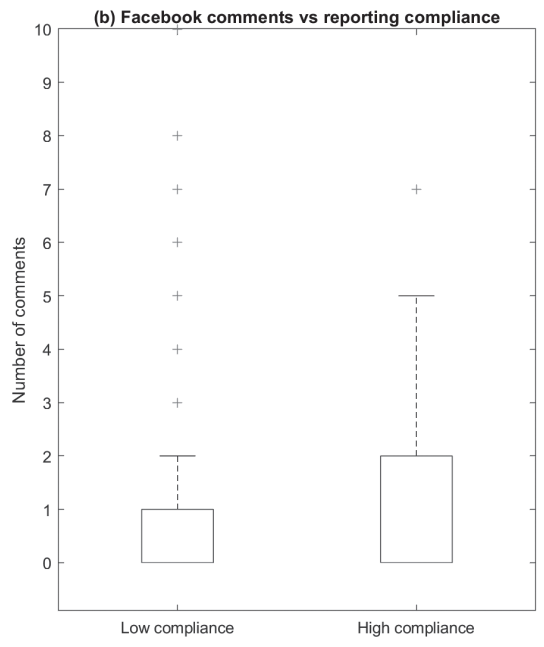

Figure 3. Box plots showing the social media responses to articles with low versus high compliance with reporting guidelines. (a) Sharing on Facebook. (b) Comments on Facebook. Y-axes are truncated, with not all outliers shown. word suicide in the headline; $n=157 ; 57.1 \%$ ) and highest with ninth guideline (promotion of public memorials; $n=263 ; 95.6 \%)$. Articles were compliant with a median of seven recommendations (range $=3-9$; see Figure $2 b$ ) articles that complied with eight or nine recommendations were therefore considered to have high compliance.

Articles that were highly compliant with the reporting guidelines were shared on Facebook more frequently than those with low compliance (median $=93$ vs. 38 shares, $p=.017$; see Figure 3). The median number of comments in both the low- and high-compliance group was 0 , which was not a significant difference $(p=.340)$.

\section{Discussion}

Overall, this study found a good degree of compliance with responsible media reporting guidelines, with only a small number of articles being compliant with as few as three of the nine recommendations. The lowest compliance was found in relation to the use of suicide in the headline, suggesting this is still a relatively common means for attracting readers' attention. The provision of help-seeking information was associated with the second lowest degree of compliance, which is surprising given the ready availability of information on crisis support services in Australia.

Highly compliant media articles were found to be shared more often on Facebook than less compliant articles, and while this association disappeared when number of comments was examined, it was likely due to a relatively low number of comments overall.

It was also noted that the differences in social media sharing may not be solely due to compliance with the reporting guidelines. It may also be the case that more compliant articles were published by larger, more reputational organizations with a higher standard of reporting, and potentially a higher likelihood that journalists have been trained in the guidelines. Other factors associated with the article (including celebrity status, individual writers and 
news offices, and specific communities or regionality) are likely to be important drivers of online engagement.

\section{Limitations}

There were a number of limitations in the present study. It is possible that there were differences in the nature of the comments, where an article with stigmatizing or sensationalist language may have led to more stigmatizing comments online, for example. Future additional data collection and coding of comment text may help address this issue. The present analysis focused on online engagement on the Facebook platform due to the availability of structured data through the API, and other online platforms (including social media platforms and news comment sections) may reveal different patterns of engagement. It is also possible that content may have been syndicated across multiple publications, and therefore appeared multiple times within the analysis dataset - however, a post hoc examination found no duplication of headlines. Analysis was conducted on the basis of the number of Mindframe recommendations followed, and assumed all recommendations are weighted equally - no attempt was made to prioritize or weight individual recommendations based on importance or impact. Finally, it is acknowledged that this is an observational study, and causal inferences cannot be made. It should therefore not be concluded that improving reporting compliance will increase online engagement.

\section{Conclusion}

The current study indicates that there is reasonably good compliance among Australian online media articles with the Mindframe suicide reporting guidelines, although compliance varied across individual recommendations. Overall, compliant news articles were shared more often on social media. Understanding how to maximize the dissemination of positive messaging and stories may help minimize the risk of contagion from reporting of suicide events.

\section{References}

Australian Bureau of Statistics. (2019). 3303.0 - causes of death, Australia, 2018. Canberra, Australia: Author.

Colombo, G. B., Burnap, P., Hodorog, A., \& Scourfield, J. (2016). Analysing the connectivity and communication of suicidal users on twitter. Computer Communications, 73(Pt B), 291-300. https:// doi.org/10.1016/j.comcom.2015.07.018
De Choudhury, M., \& Kiciman, E. (2017). The language of social support in social media and its effect on suicidal ideation risk. Proceedings of the International AAAl Conference on Weblogs and Social Media, 2017, 32-41.

Department of Health. (2017). The fifth national mental health and suicide prevention plan. Canberra, Australia: Commonwealth of Australia.

Everymind. (2014). Reporting suicide and mental illness: A Mindframe resource for media professionals. Newcastle, Australia: Everymind.

Gould, M. S., Kleinman, M. H., Lake, A. M., Forman, J., \& Midle, J. B. (2014). Newspaper coverage of suicide and initiation of suicide clusters in teenagers in the USA, 1988-96: A retrospective, population-based, case-control study. Lancet Psychiatry, 1(1), 34-43. https://doi.org/10.1016/S2215-0366(14)70225-1

Lee, J., Lee, W. Y., Hwang, J. S., \& Stack, S. J. (2014). To what extent does the reporting behavior of the media regarding a celebrity suicide influence subsequent suicides in South Korea? Suicide and Life-Threatening Behavior, 44(4), 457-472. https://doi. org/10.1111/sltb.12109

Mueller, A. S., \& Abrutyn, S. (2015). Suicidal disclosures among friends: using social network data to understand suicide contagion. Journal of Health and Social Behavior, 56(1), 131-148. https://doi.org/10.1177/0022146514568793

Niederkrotenthaler, T., Fu, K. W., Yip, P. S., Fong, D. Y., Stack, S., Cheng, Q., \& Pirkis, J. (2012). Changes in suicide rates following media reports on celebrity suicide: A meta-analysis. J Epidemiol Community Health, 66(11), 1037-1042. https://doi.org/10.1136/ jech-2011-200707

Niederkrotenthaler, T., Voracek, M., Herberth, A., Till, B., Strauss, M., Etzersdorfer, E., ... Sonneck, G. (2010). Role of media reports in completed and prevented suicide: Werther v. Papageno effects. Br J Psychiatry, 197(3), 234-243. https://doi.org/10.1192/ bjp.bp. 109.074633

Pirkis, J., \& Blood, W. (2010). Suicide and the news and information media: A critical review. Canberra, Australia: Commonwealth of Australia.

World Health Organization and the International Association for Suicide Prevention. (2017). Preventing suicide: A resource for media professionals - update 2017. Geneva, Switzerland: Author.

\section{History}

Received January 16, 2020

Revision received April 26, 2020

Accepted May 1, 2020

Published online October 9, 2020

\section{Funding}

This work was supported by the NHMRC Centre of Research Excellence in Suicide Prevention (APP1152952). MT was supported by a NHMRC Early Career Fellowship (APP1138710).

\author{
Mark Larsen \\ Black Dog Institute \\ Hospital Road \\ Randwick, NSW 2031 \\ Australia \\ mark.larsen@blackdog.org.au
}

Tiana Edwards is a final-year medical student at UNSW Sydney, working with the Black Dog Institute, UNSW Sydney, Australia. She has interests in psychiatry, mental wellbeing, and functional disorders. This work formed the research component of her undergraduate degree. 
Michelle Torok, PhD, is a senior research fellow with the Black Dog Institute, UNSW Sydney, Australia, and a NHMRC early career fellow. She leads of program of research in suicide prevention, involving digital technologies, and translational science at scale. As part of this, coleads a multilevel suicide prevention trial known as LifeSpan.

Lauren McGillivray, PhD, is a postdoctoral research fellow with the Black Dog Institute, UNSW Sydney, Australia. Her research evaluates the effectiveness of a mental health promotion and suicide prevention program for secondary school students, and examines a mHealth intervention to help young people manage suicidal thoughts.

Trinn Ford is a graduate of law and criminology and criminal justice at UNSW Sydney, Australia. They were formerly a research assistant with UNSW Law, and are now a research officer for the Royal Commission into Violence, Abuse, Neglect and Exploitation of People with Disability.
Katherine Mok, PhD, is a postdoctoral research fellow with the Black Dog Institute, UNSW Sydney, Australia. She is working on a large-scale, multilevel intervention for suicide prevention trial. Her research examines the relationship between suicide and the media, suicide-related internet use in young people, and community-based suicide prevention.

Emily Li, PhD, was formerly a research officer with the Black Dog Institute, UNSW Sydney, and is now a Project Officer with St. Vincent's Hospital Sydney, Australia. Her research interests include the application of mHealth interventions to suicide prevention and alcohol and other drug use.

Mark Larsen, DPhil, is a senior research fellow with the Black Dog Institute, UNSW Sydney, Australia. His research examines the application of digital health approaches to suicide prevention, and he leads the Means Restriction and Public Safety stream of the NHMRC Centre of Research Excellence in Suicide Prevention. 